\title{
Specific Relationship between the Shape of the Readiness Potential, Subjective Decision Time, and Waiting Time Predicted by an Accumulator Model with Temporally Autocorrelated Input Noise
}

\author{
(D) Aaron Schurger ${ }^{1,2}$ \\ DOI:http://dx.doi.org/10.1523/ENEURO.0302-17.2018 \\ ${ }^{1}$ Cognitive Neuroimaging Unit, INSERM, Gif sur Yvette 91191, France and ${ }^{2}$ Commissariat à l'Energie Atomique, \\ NeuroSpin Center, Gif sur Yvette 91191, France
}

\begin{abstract}
Self-initiated movements are reliably preceded by a gradual buildup of neuronal activity known as the readiness potential (RP). Recent evidence suggests that the RP may reflect subthreshold stochastic fluctuations in neural activity that can be modeled as a process of accumulation to bound. One element of accumulator models that has been largely overlooked in the literature is the stochastic term, which is traditionally modeled as Gaussian white noise. While there may be practical reasons for this choice, we have long known that noise in neural systems is not white - it is long-term correlated with spectral density of the form $1 / f^{\beta}$ (with roughly $1<\beta<3$ ) across a broad range of spatial scales. I explored the behavior of a leaky stochastic accumulator when the noise over which it accumulates is temporally autocorrelated. I also allowed for the possibility that the RP, as measured at the scalp, might reflect the input to the accumulator (i.e., its stochastic noise component) rather than its output. These two premises led to two novel predictions that I empirically confirmed on behavioral and electroencephalography data from human subjects performing a self-initiated movement task. In addition to generating these two predictions, the model also suggested biologically plausible levels of autocorrelation, consistent with the degree of autocorrelation in our empirical data and in prior reports. These results expose new perspectives for accumulator models by suggesting that the spectral properties of the stochastic input should be allowed to vary, consistent with the nature of biological neural noise.
\end{abstract}

Key words: autocorrelation; Bereitschaftspotential; leaky stochastic accumulator; readiness potential; voluntary action

\section{Significance Statement}

The cortical readiness potential (RP) is a gradual buildup of scalp electrical potential, and underlying neural activity in motor areas, that reliably precedes the onset of voluntary self-initiated movements by up to one second or more. More than fifty years after its discovery, the functional nature of the RP remains unclear. Here I argue, based on empirical evidence, that the RP reflects the stochastic input to an accumulation-to-bound decision process, and that this stochastic input is temporally autocorrelated, and not Gaussian white noise as it is traditionally modeled. The argument is supported by testing and confirming two novel predictions that emerge from an accumulator model when the stochastic input noise is autocorrelated rather than white.

\section{Introduction}

Uncued voluntary movements are preceded reliably by a slow buildup of cortical activity known as the Bere-

Received August 30, 2017; accepted January 18, 2018; First published January 23, 2018.

The authors declare no competing financial interests. itschaftspotential or readiness potential (RP; Shibasaki and Hallett, 2006). Since its discovery in the 1960s (Kornhuber and Deecke, 1965), the RP has been inter- 
preted as a sign of movement preparation, the outcome of a preconscious neural decision to initiate an action (Libet et al., 1983). Recent evidence, however, supports a different interpretation: the RP reflects ongoing stochastic fluctuations in neural activity that favor the spontaneous emission of a movement at certain times more so than at others (Schurger et al., 2012; Murakami et al., 2014; Schmidt et al., 2016). Both the shape of the $\mathrm{RP}$ and the distribution of waiting times (how long the subject waits before producing a spontaneous movement) can be well described by a leaky stochastic accumulator model (Schurger et al., 2012; Murakami et al., 2014).

Accumulator models at a minimum consist of a constant term (reflecting the decision evidence) plus a stochastic term (see Materials and Methods). Integration over these "inputs" to the accumulator results in the "output" of the accumulator, commonly referred to as the decision variable because a decision is made and action initiated when the output exceeds a certain threshold. One element of accumulator models that has been largely overlooked in the literature is the stochastic term, which is traditionally modeled as Gaussian white noise. While there may be practical reasons for this assumption, it is well known that noise in neural systems is not white, but instead is pink - it is long-term correlated with spectral density of the form $1 / f^{\beta}$ (with roughly $1<\beta<3 ; \beta=0$ for white noise) across a broad range of spatial scales (Fig. 1; Pereda et al., 1998; He et al., 2010). Whether or not accumulator models should account for the spectral properties of neural noise is an open question. At the same time, we can also ask whether or not the stochastic term in such models can be mapped onto a well-defined neural phenomenon.

A recent study found evidence for an accumulator process in area M2 of rats (homolog of human premotor cortex) performing a task where the rat could spontaneously abandon waiting for a large reward and instead opt for an immediate and certain, but smaller reward (Murakami et al., 2014). Using single-unit recordings, these authors were able to identify two different and interspersed functional subtypes of neurons: ones that behaved like the inputs to an accumulator, with tonic firing rates proportional to the length of time that the rat waited, and others that behaved like the outputs of an accumulator, with firing rates ramping up to a fixed threshold level just when the rat withdrew from the waiting station and sought out the small but certain reward. Which of these two is more likely to dominate in signals picked up by a

\footnotetext{
640626.

Acknowledgements: I thank Jacobo Sitt and Philippe Ciuciu for contributing Matlab code.

Correspondence should be addressed to Aaron Schurger at the above address, E-mail: aaron.schurger@gmail.com.

DOI:http://dx.doi.org/10.1523/ENEURO.0302-17.2018

Copyright (C) 2018 Schurger

This is an open-access article distributed under the terms of the Creative Commons Attribution 4.0 International license, which permits unrestricted use, distribution and reproduction in any medium provided that the original work is properly attributed.
}

scalp electrode, the vantage point from which the RP is commonly measured, is unknown.

Thus, here I take up two distinct but related points: (1) that accumulator models of decision-making should allow the slope $(\beta)$ of the noise spectrum to vary instead of using white noise (Fig. 1), especially when the imperative (drift term) is weak relative to the noise; and (2) that a buildup in event-preceding EEG potential might reflect the average stochastic input to an accumulator process ("RP-as-input") rather than its average output ("RP-as-output").

With the above two points in mind, I explored the behavior of a leaky stochastic accumulator when the noise over which it accumulated was temporally autocorrelated, and empirically tested two novel predictions that emerged. I tested these two predictions on behavioral and EEG data recorded while subjects performed Libet's selfinitiated movement task (Libet et al., 1983). One prediction was that the shape of the RP should vary in a specific way as a function of the waiting time (how long the subject waits before producing a spontaneous movement). The second prediction concerned the subjective estimate of the time of the "urge" to move, what Libet et al. (1983) referred to as 'W' time: $W$ time was predicted to vary as a function of waiting time, becoming earlier (with respect to movement onset) with longer waiting times. Both predictions were confirmed, with the model suggesting biologically plausible levels of autocorrelation $(\beta \cong 1.4)$. Taken together, the results suggest that the EEG RP may reflect the autocorrelated stochastic input to an accumulationto-bound process, and highlight the potential utility of using biologically realistic noise in accumulation-tobound models.

\section{Materials and Methods}

I reanalyzed behavioral and EEG data from a prior study (Schurger et al., 2012). Details about human subjects, stimuli and task, and data preprocessing are summarized below. Details about data analyses, statistics, and computational modeling are reported in full.

\section{Human subjects}

A total of 16 subjects participated in the study (six female, mean age 28, one left handed). Subjects were paid for their participation and all gave written informed consent. Two of the subjects did not exhibit a RP (a negative trend in electrical potential preceding movement onset at $\mathrm{Cz}$ or any adjacent electrode) and so were excluded from further analyses, leaving $N=14$.

\section{Stimuli and task}

Subjects sat $\sim 60 \mathrm{~cm}$ in front of a translucent screen onto which the stimulus was back-projected. The subject sat in a reclined position with dim ambient lighting. The stimulus was a small clock face (white on a black background) with a diameter of $\sim 6^{\circ}$ of viewing angle. A small white dot circled smoothly around the edge of the clock dial, completing one full cycle every $3 \mathrm{~s}$ (equivalent to 50 ms per tick mark on the clock). Each trial began with the onset of the clock rotation. Subjects were instructed to wait for the clock to complete one full cycle. After that the 
A
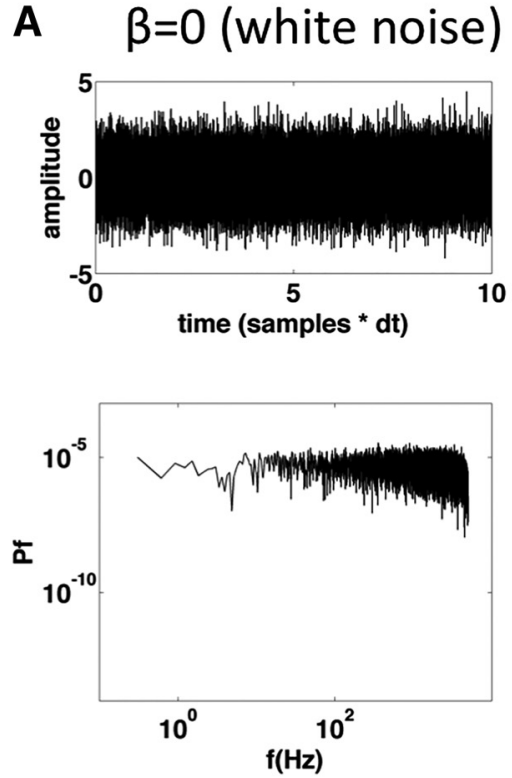

D
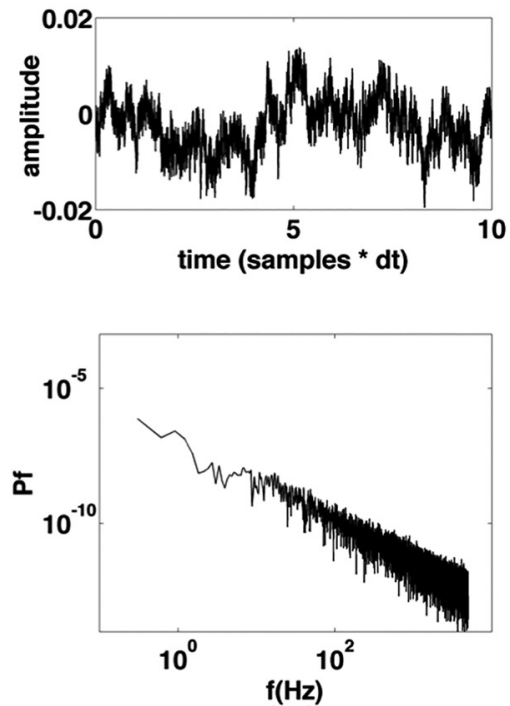

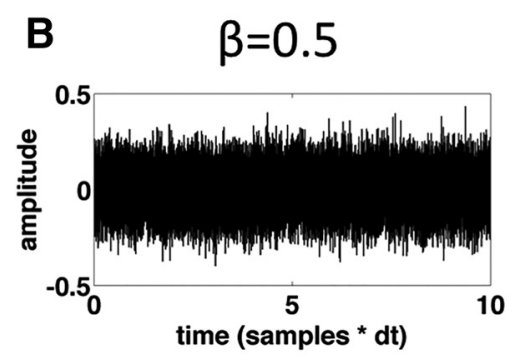

C

$\beta=1.0$
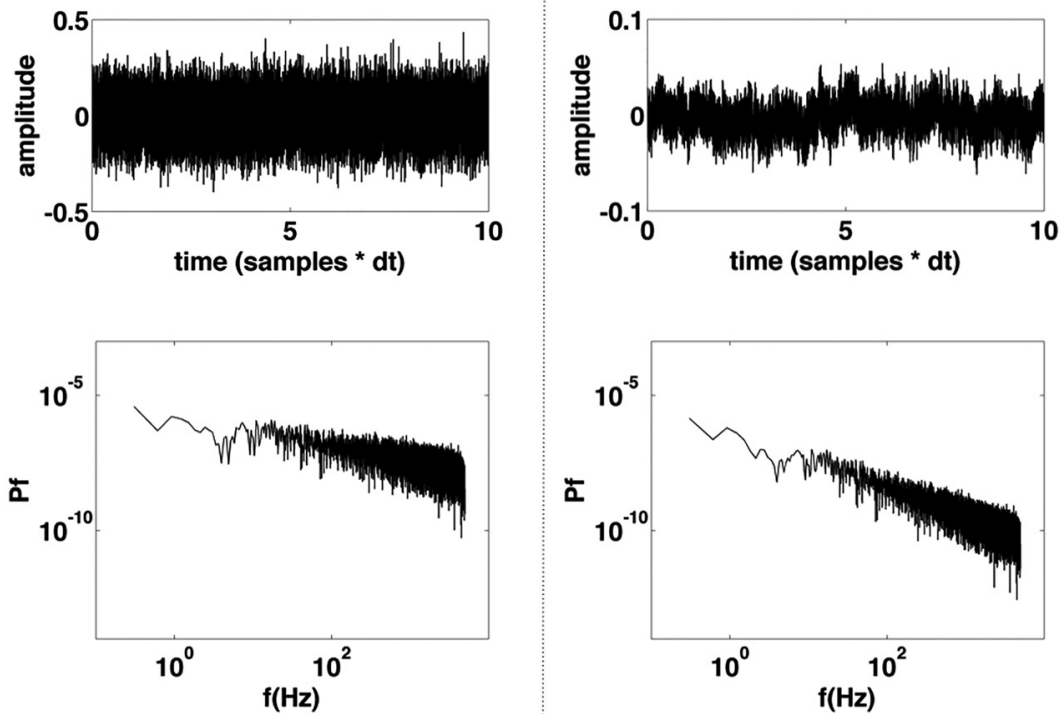

F lowpass filtered
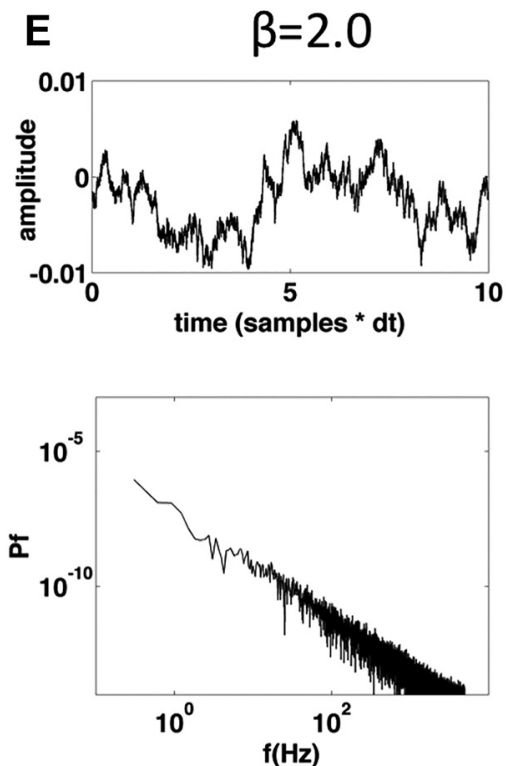
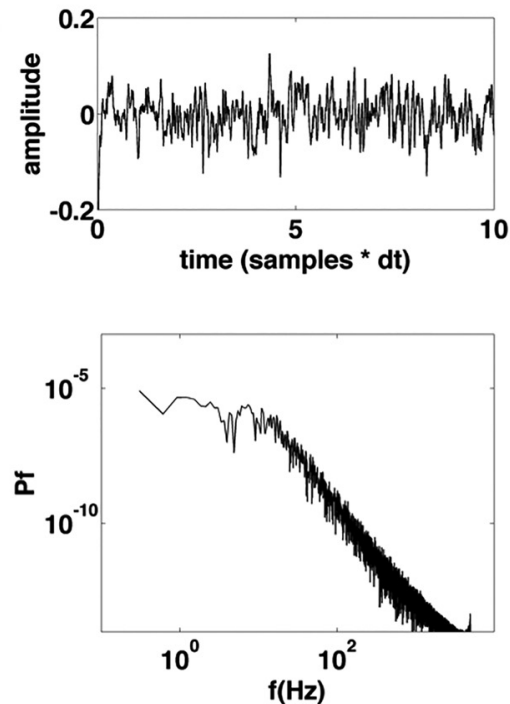

Figure 1. Illustration of white noise and different flavors of pink noise. $\boldsymbol{A}-\boldsymbol{E}$, Noise with different $1 / f$ exponents $(\beta$, where spectral power scales as $1 / f^{\beta}$ in log-log coordinates). The upper axis of each panel shows the time series and the lower axis shows the power spectrum; $\beta$ specifies the slope of the power spectrum in units of log power versus log frequency, so if $\beta=0$, then the noise is white (flat power spectrum, roughly equal power in all frequencies, each sample independent of all other samples). Notice that as $\beta$ increases, the time series becomes more and more dominated by low-frequency fluctuations, as indicated by the slope of the spectra. Time series are shown for values of $\beta$ between 0 and 2 to illustrate the way that time series change qualitatively as $\beta$ changes. $\boldsymbol{F}$, Same noise series as in A after lowpass filtering with a first-order Butterworth filter (cutoff frequency of $1 \mathrm{~Hz}$ ).

subject was free to perform the instructed movement (pressing a button with the thumb of the dominant hand) at any time. Subjects were encouraged to try not to preplan the moment when they would press the button, but rather to do so spontaneously without any forethought (Libet et al., 1983). After pressing the button, the dot continued to circle the clock face for one more second and then the screen went blank. Subjects were instructed that after the screen went blank they should report (verbally) what had been the position of the dot at the moment that they first became aware of their urge to press the button. In addition to this task (the "classic" task) subjects also performed a second task which was identical except that on some trials subjects were interrupted by an auditory "click" indicating that they should press the button immediately (the "interruptus" task). The analyses re- 
ported here were performed on a combination of the data form the first task and the uninterrupted trials from the second task.

\section{EEG data acquisition}

EEG data were recorded inside of a shielded room using a 60-channel EEG system (Elekta NeuroMag EEG/ MEG system) sampled at $1000 \mathrm{~Hz}$. Of the 60 channels, I used a subset encompassing the standard 10-20 montage, with the addition of electrodes $\mathrm{C} 1, \mathrm{C} 2, \mathrm{FC} 1$, and FC2.

\section{EEG data preprocessing}

Data preprocessing and analysis were performed using Matlab (MathWorks Inc) along with the FieldTrip toolbox for Matlab (Oostenveld et al., 2011). Data were first downsampled to $250 \mathrm{~Hz}$. Independent component analysis (ICA) was used to identify and remove ocular artifacts from the data (Jung et al., 2000), and trials with artifacts remaining after this step were excluded by visual inspection. I extracted data epochs time locked to the first button press after the start of the trial. Each data epoch covered the window of time extending from -3.5 to +1.0 $s$ relative to the button press.

\section{Data analyses and statistics \\ Behavioral}

I define the "waiting time" (or just "wait time") as the amount of time, in seconds, that elapsed from the beginning of the trial until the subject first pressed the button. 'W' time (Libet et al., 1983) was the time, relative to the onset of the button press, that the subject reported first having been aware of the urge or decision to press the button. This was recorded by taking the clock time at which the subject reported having had the urge and subtracting it from the clock time of the actual movement. The correlation between waiting time and $\mathrm{W}$ time was computed using Pearson's correlation coefficient applied both to the pooled data from all subjects and also separately for each subject. In the latter case, the resulting $r$ values across subjects were tested for significance (difference from zero) using Wilcoxon's signed rank test.

\section{EEG}

EEG data epochs were sorted according to waiting time into the lower 33rd percentile (short waiting time) and the upper 33rd percentile (long waiting time), and averaged together within each group to compare the shape of the $\mathrm{RP}$ for short and long waiting times. Differences in the amplitude of the RP at each time point were tested for significance using a signed rank test and then subjected to a cluster-based permutation test to correct for multiple comparisons. Unless specified otherwise, the RP was measured at electrode $\mathrm{C} 1$ (C2 if left handed).

\section{Leaky stochastic accumulator model}

Accumulator models at a minimum involve integration over an input signal (drift or "imperative") plus Gaussian white noise. Noise in the brain, however, is temporally autocorrelated with spectral density of the form $1 / f^{\beta}$ with typically $1<\beta<3$ ("pink" noise). The model that I used was the same as that used in prior studies (Schurger et al.,
2012; Murakami et al., 2014), namely the leaky stochastic accumulator model (Usher and McClelland, 2001), except that the noise was pink, with $\beta$ allowed to vary arbitrarily, instead of white ( $\beta$ fixed at 0 ). Performing a run of the simulation amounts to performing numerical integration over the following differential equation:

$$
d x=(I-k x) d t+c \xi_{\beta} \sqrt{d t}
$$

where $I$ is the drift rate (the imperative to move), $k$ is leak, and $\xi_{\beta}$ is noise with $1 / f$ exponent $\beta$ allowed to vary arbitrarily, from 0 (white noise) to $3 ; c$ is a noise scaling factor (by convention $c=0.1$ ). I sometimes refer to the output of the accumulator ( $x$ in the equation above) as the decision variable. After each run of the simulation I extracted two "data epochs," one from the noise input to the accumulator and one from the output of the accumulator. Both epochs were time aligned to the sample at which the output of the accumulator first crossed the threshold, and spanned the interval from 5000 samples before the crossing to 500 samples after. When I fit the event-locked input to the accumulator to the shape of the RP, I use the term RP-as-input, and when I fit the event-locked output of the accumulator to the shape of the RP, I use the term $\mathrm{RP}$-as-output. The simulation is one and the same in both cases, the only difference being which by-product of the simulation (the input or the output) is considered to represent the RP.

\section{Generation of pink noise}

Normally, when simulating an accumulator process, a series of computer-generated Gaussian-distributed pseudorandom numbers is used to instantiate the (white) noise term. For optimal performance it is best to generate the entire time series of pseudo-random numbers all at once at the beginning of the simulation, and then step through it, rather than generating a single random number on each iteration. Having the entire time course of the noise in hand allows one to change the spectral properties of the noise as desired. Power-law or $1 / f$ noise has spectral power that is inversely proportional to frequency, roughly following a negative-sloping line in log-log coordinates (He et al., 2010; Fig. 1): $P \propto 1 / f^{\beta}$ with $\beta$ commonly referred to as the "1/f exponent." For the simulations reported here, I first generated a time series of Gaussian pseudorandom numbers and then altered the spectrum of this noise as follows: first the signal was converted into the frequency domain via a fast Fourier transform (fft in Matlab); then a given (negative) slope was imposed on the power spectrum by multiplication with a log-linear function having the desired slope; and then the signal was converted back into the time domain via an inverse Fourier transform (ifft in Matlab). The resulting time series, which could have any arbitrary $\beta$ (for examples, see Fig. 1 ), was then used as the noise input to the simulated accumulator process. Note that I make no assumptions or claims about the spectral properties of the time series other than that they must be temporally autocorrelated. The procedure described above is simply a convenient way to obtain autocorrelated noise while 
parametrically varying the degree of temporal autocorrelation in the time series.

\section{Data fitting}

For separate runs of the simulation, the average time course of the model output or the stochastic input to the model was fit to the average RP across subjects. The time courses were time locked to the first crossing time, which is of course always determined based on the output of the accumulator. For both the RP-as-input and RP-as-output simulations the fitting procedure included a scaling factor whereby the amplitude of the simulated RP was scaled to that of the empirical RP. In each case, the normalized distribution of first-crossing times of the model was simultaneously fit to the normalized empirical waiting time distribution. Fitting was performed using multidimensional unconstrained nonlinear minimization (Nelder-Mead; function fminsearch in Matlab) on the mean-squared error between the simulated and empirical data. Appropriate starting values for the parameters, including scaling parameters, were identified by first trying a few fits by hand.

\section{Modeling of ' $W$ ' time and derivation of the prediction}

I modeled the subjective estimate of the time of the conscious urge to move (Libet's ' $W$ ' time) by adding a second threshold, slightly lower than the main activation threshold (Fig. 2), similar to Ganos et al. (2015). This "advance warning" threshold is sufficiently close to the main threshold that crossing it is a good predictor that the main threshold is about to be crossed (i.e., movement is about to ensue) with very high probability. 'W' time in this model is simply the temporal delay between the crossing of the two thresholds, expressed with respect to the crossing of the second threshold (i.e., a negative number). Note that I do not assume that this predictive information uniquely determines ' $W$ ' time, but only that it informs ' $W$ ' time. Prior evidence indicates that neural information from both before and after movement onset can influence ' $W$ ' time (Sirigu et al., 2004; Lau et al., 2007; Banks and Isham, 2009; Douglas et al., 2015), and thus that the brain likely makes use of information from both before and after movement onset in its estimation of 'W' time. Here, I only assume that a premovement prediction about an upcoming movement and its reafferent consequences (Wolpert, 1997 ) is at least part of the information that contributes to the brain's estimate of ' $W$ ' time.

\section{Estimation of the $1 /$ fexponent $(\beta)$}

The $1 / f$ exponent was computed as the sign-reversed slope of the best fitting line fit to the fractal component of the power spectrum in log-log coordinates. The fractal component of the power spectrum was estimated using the IRASA method of Wen and Liu (2016) and the best fitting line was estimated using ordinary least squares regression (Iscov in Matlab).

\section{Code accessibility}

Computer code (Matlab) for running the simulations is available as Extended Data 1, and is also available online at https://bitbucket.org/aschurger/lsa_rp_model.

\section{Results}

\section{Behavioral}

Waiting time

Subjects waited on average 7.1 ( $\pm 0.63 \mathrm{~s}$ SEM) seconds to produce a movement in the classic Libet task, and 5.4 $\mathrm{s}( \pm 0.31 \mathrm{~s}$ SEM) in the interruptus task. This difference was significant $(p<0.001$; two-tailed $t$ test, $t=4.5$, df $=$ 14), although the two distributions were mostly overlapping (see Fig. 3B of Schurger et al., 2012). Since I combined noninterrupted trials from the interruptus task with data from the classic task, I performed an additional test (see below) to make sure that this average difference in waiting time did not account for any of the other results related to waiting time.

\section{'W' time}

Libet et al. (1983) used the term 'W' time to refer to the subjective estimate of the time of the conscious urge to move. The mean ' $W$ ' time in the classic task was $-142 \mathrm{~ms}$ ( $\pm 34 \mathrm{~ms}$ SEM), and the mean ' $W$ ' time in the interruptus task was $-126 \mathrm{~ms}$ ( $\pm 44 \mathrm{~ms}$ SEM). The ' $W$ ' times in the two tasks were not significantly different across subjects $(p=$ 0.45; two-sided paired samples signed rank test).

\section{EEG RP}

Fourteen of 16 subjects exhibited the gradual negative deflection in scalp electrical potential before movement onset, at or near the vertex, that is characteristic of the RP. The two subjects that did not exhibit an RP were excluded from further analyses. The average RP across subjects $(N=14)$ is shown in Figure $3 B$. The tail of the RP extended back in time at least two full seconds before nearing its horizontal asymptote.

\section{Data fitting}

The RP-as-output variant of the model has been shown previously to fit the data well (Schurger et al., 2012), but what about the RP-as-input variant? Although the present argument is focused on empirical predictions made by the two models, nevertheless it was important to confirm that the RP-as-input model was capable of fitting the data, before going on to test empirical predictions derived from it.

The RP-as-input model is in fact capable of producing a very good fit to the data. Figure 3 shows the normalized distribution of first crossing times of the output of the accumulator fit to the normalized empirical waiting time distribution $(n=14)$. Also shown is the average time course of the stochastic input to the accumulator (time locked to threshold crossings in the output), fit to the average RP. The parameters for the best fit were obtained after an exhaustive search of the parameter space, and were as follows: $\beta=1.4, \mathrm{I}=0.1, \mathrm{k}=0.6$, and threshold $=0.1256$. Thus, a single set of parameters exists such that the RP is well fit by the event-locked stochastic input to the accumulator and the waiting time distribution is well fit by the first-crossing time distribution of the output. It is noteworthy that the value for $\beta$ was 1.4 , which is approximately the value for $\beta$ found in our EEG data (1.34 \pm 0.14 SEM; Fig. 4) and that has been observed in the past for EEG data (Pereda et al., 1998; reported $\beta=1.5$ ). 


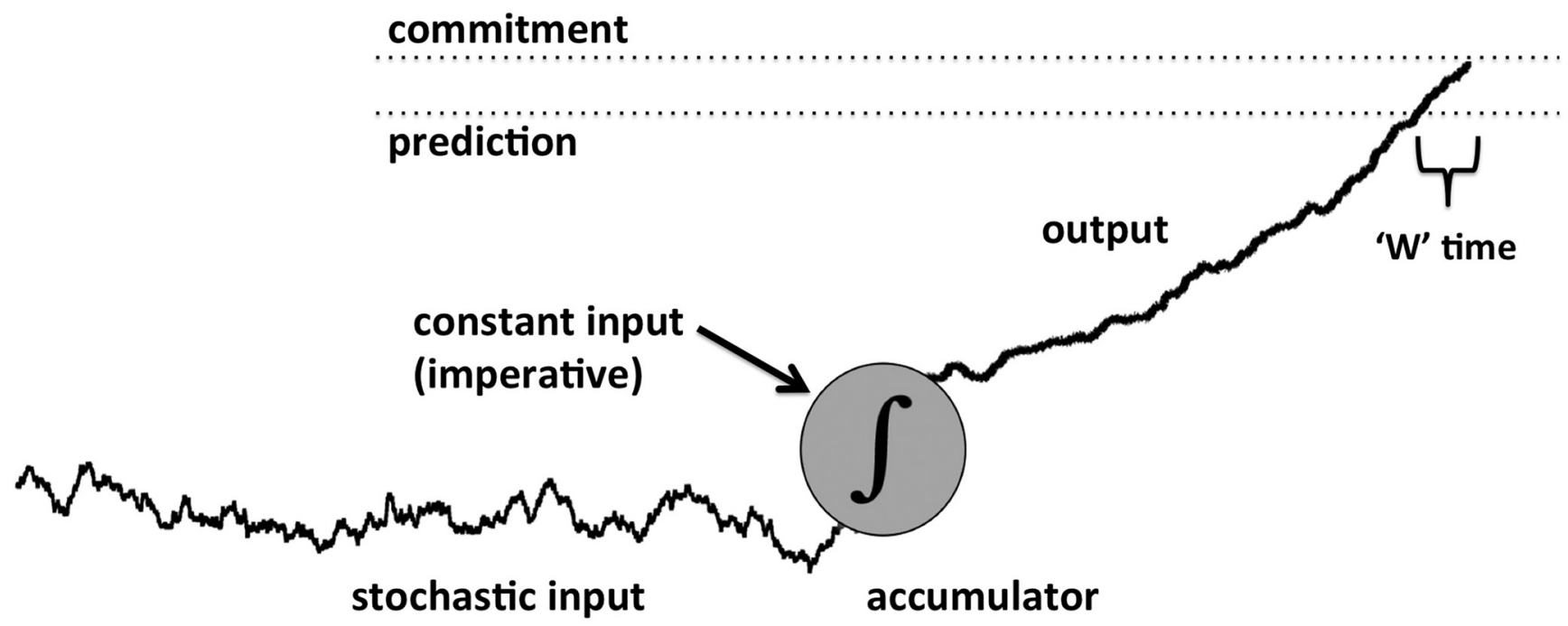

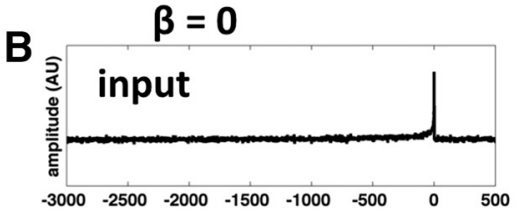

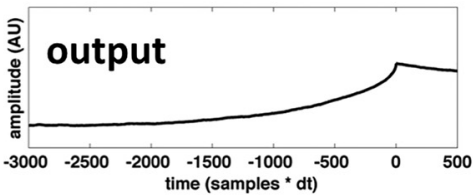

$\beta=1.5$
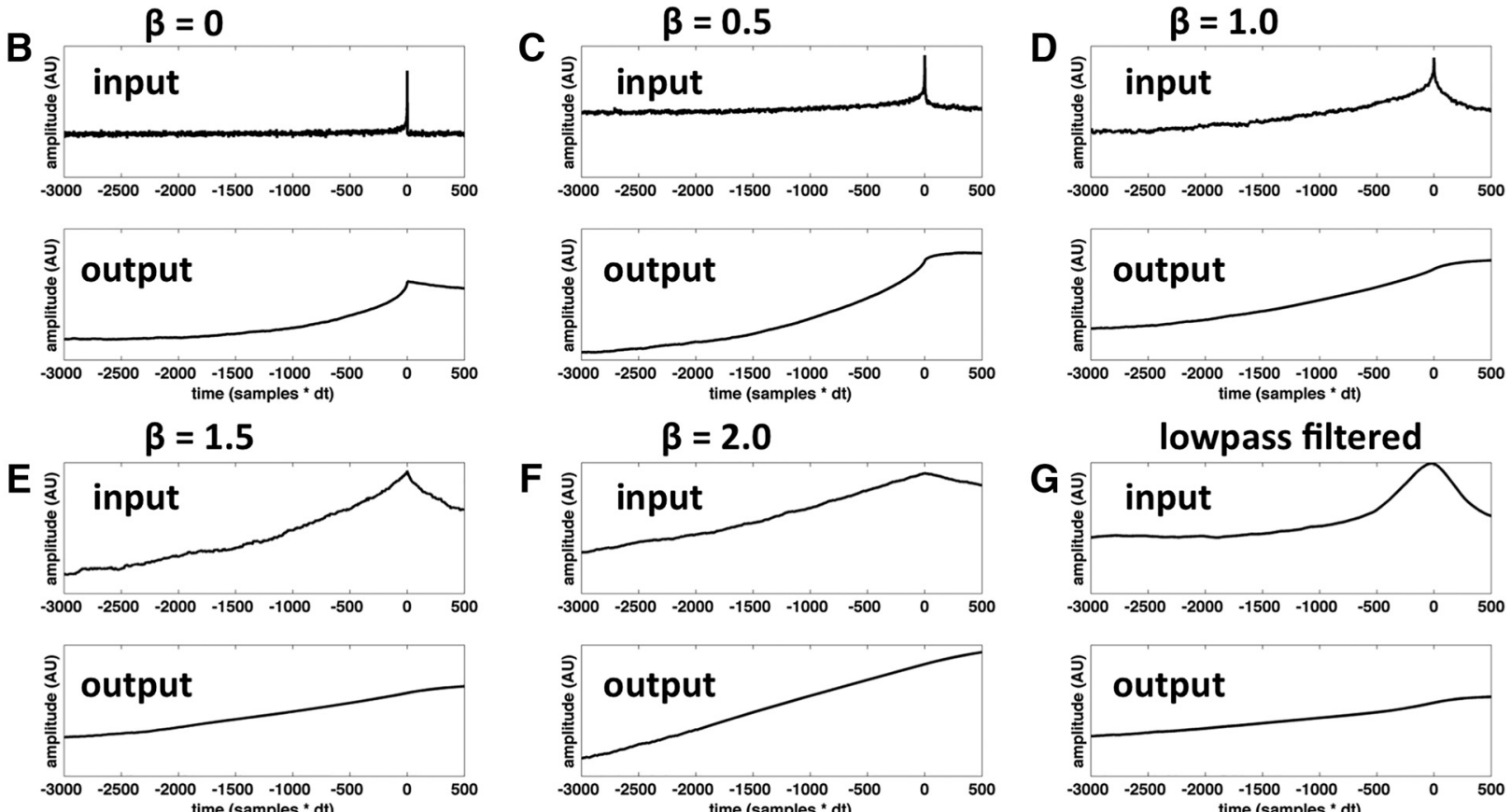

$$
\boldsymbol{\beta}=\mathbf{2 . 0}
$$
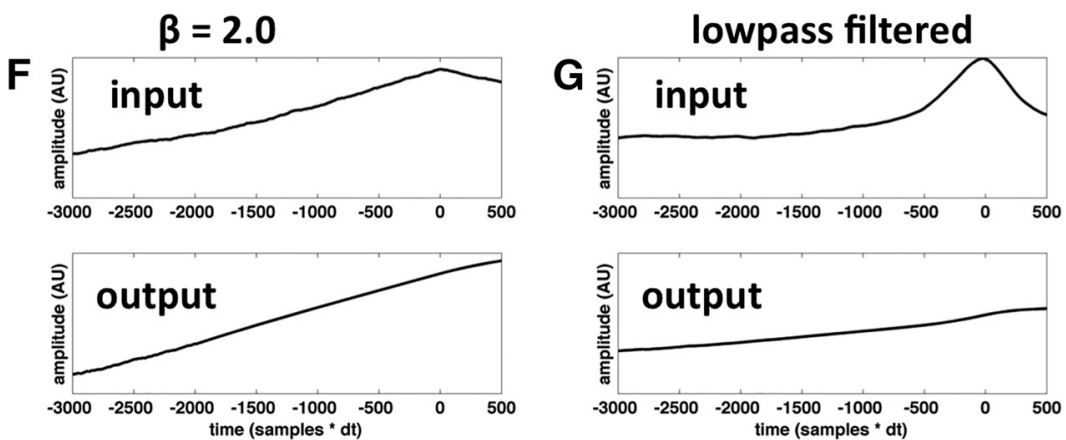

Figure 2. Schematic illustration of the model $(\boldsymbol{A})$ with sample input and output $(\boldsymbol{B}-\mathbf{G})$. In $\boldsymbol{A}$, the $\int$ symbol represents the neural accumulator, with pink noise plus constant input on the left and output on the right. The higher of the two thresholds is the activation threshold: when this threshold is crossed, then the "neural decision to move" has been made and movement ensues. The lower of the two thresholds is a self-monitoring threshold: when this threshold is crossed a signal is generated indicating that movement is about to ensue with very high probability. When subjects are asked to estimate the time at which they were first aware of an urge to move ('W' time), this decision is (according to the model) informed by the delay between the crossing of the two thresholds. $\boldsymbol{B}-\boldsymbol{F}$, Average input and output from the model for different values of the $1 / f$ exponent $(\beta)$. $\boldsymbol{G}$, Same for lowpass filtered white noise (see Materials and Methods).

One might ask whether or not it is necessary to use $1 / f$ noise as input to the accumulator. Perhaps all that is necessary is to suppress the higher frequencies, in which case a low-pass filter applied to white noise might suffice (Fig. 1F). One problem with this reasoning is that this procedure could be seen as simply a very crude way of approximating $1 / f$ noise, which would bring us back to the original question. Nevertheless, it is unclear a-priori how well the model would fit the data using low-pass filtered white noise instead of $1 / f$ noise as input to the accumulator. I tested this possibility using a first-order Butterworth low-pass filter, varying the cutoff frequency. I used 

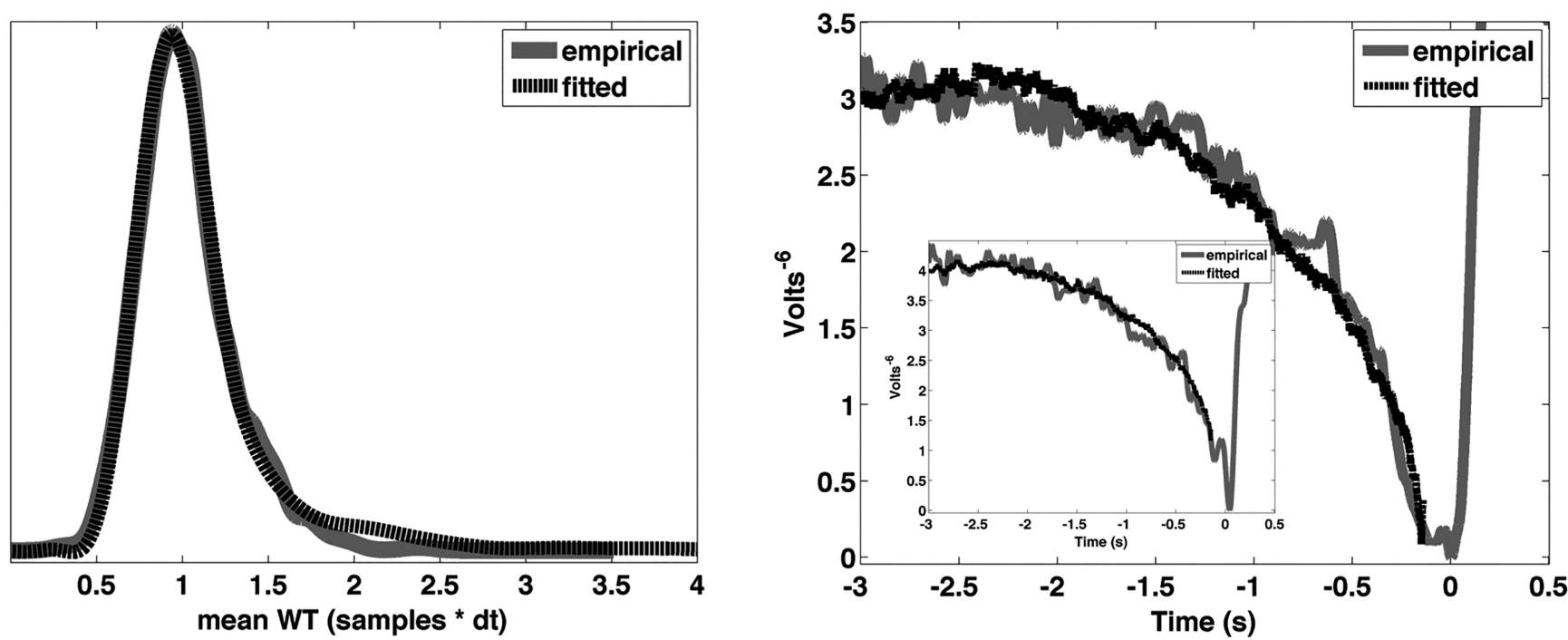

Figure 3. Fitting the model to the data under the RP-as-input interpretation. Left, The distribution of first crossing times (dashed black line) fit to the empirical distribution of waiting times (solid gray line). Right, The (sign reversed) average stochastic input to the accumulator time locked to threshold crossings in the output (dashed black line) fit to the empirical RP (RP at C1; solid gray line). The two fits were performed simultaneously, i.e., with the same parameters. The parameters used for the best fit were $\beta=1.4, I=0.1$, $\mathrm{k}=0.6$, and threshold $=0.1256$. Inset shows the same fit (same parameters), but with the RP measured at either electrode FC1, C1, or $\mathrm{Cz}$ chosen individually for each subject depending on which electrode had the highest amplitude signal at $t_{(0)}$. Note that the fitting procedure included a scaling factor whereby the amplitude of the simulated RP was scaled to that of the empirical RP.

a low-order filter because it has a shallow cutoff, thereby approximating more closely the relationship between frequency and power found in $1 / f$ noise (with higher-order filters the fit, in particular to the RP, only became worse). I used the same exhaustive parameter search here as I had used when running the model with $1 / f$ noise.

Using low-pass filtered noise as input to the accumulator, under the RP-as-input assumption, the model could fit either the RP or the wait-time distribution, at the expense of the fit to the other, but had trouble fitting both with the same set of parameters. Notably, the best fit overall using low-pass filtered noise was significantly poorer than the best fit achieved with simulated $1 / f$ noise (MSE of best fit with low-pass filtered noise $0.0271 \pm$

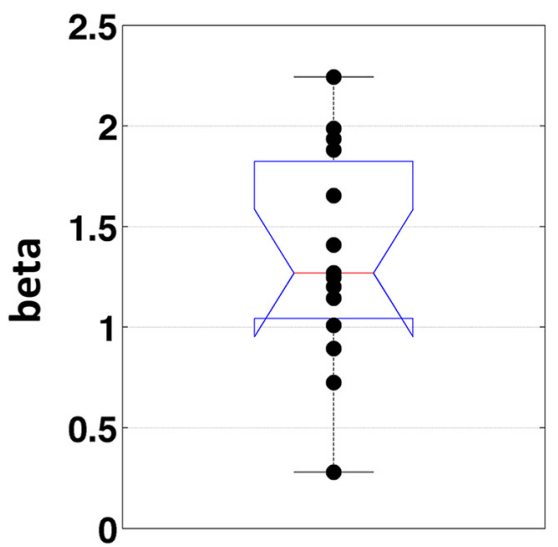

Figure 4. Estimated $1 / f$ exponent. Boxplot of the $1 / f$ exponent ( $\beta)$ estimated for each subject $(N=14)$ at electrode $C 1$. Each dot represents one subject. See Materials and Methods for the estimation procedure.
$0.00066 \mathrm{SD}$, vs $0.0146 \pm 0.00054 \mathrm{SD}$ using $1 / f$ noise; $p<$ $10^{-9}$, two-sided Wilcoxon rank sum test). Again, this might simply reflect the fact that a low-pass filter applied to white noise yields a relatively poor approximation of $1 / f$ noise. However, as stated previously, I make no claims about the necessity of "true" $1 / f$ (power-law) noise, only that the input noise should be temporally autocorrelated, although it would appear that simulated $1 / f$ noise does lead to a significantly better fit overall.

\section{Prediction 1: relationship between the shape of the RP and waiting time}

The predicted relationship between waiting time and the shape of the RP under the RP-as-input and RP-asoutput assumptions is shown in Figure 5 , for the parameters reported above $(\beta=1.4, \mathrm{I}=0.1, \mathrm{k}=0.6$, and threshold $=0.1256$ ). Although these specific parameters were the ones that resulted in the best fit under the $\mathrm{RP}$-as-input assumption, the predicted relationship between RPs for short versus long wait times remained qualitatively the same regardless of the specific parameters used, as long as $\beta$ was more than $\sim 0.5$. As illustrated in the figure, under the RP-as-input assumption the model predicts that the early RP will have a lower amplitude for long versus short waiting times (Fig. 5A). On the other hand, under the RP-as-output assumption the model predicts the opposite: a higher amplitude early RP for long versus short waiting times (Fig. 5B). The empirical data clearly support the RP-as-input interpretation (Fig. $6 ; p<$ 0.01 for the mean amplitude over the range -1.5 to $-0.5 \mathrm{~s}$, two-tailed signed rank test).

To understand the relationship (Fig. 5C,D), consider that the output of the accumulator will tend to have a steeper slope on trials with a short waiting time. This 

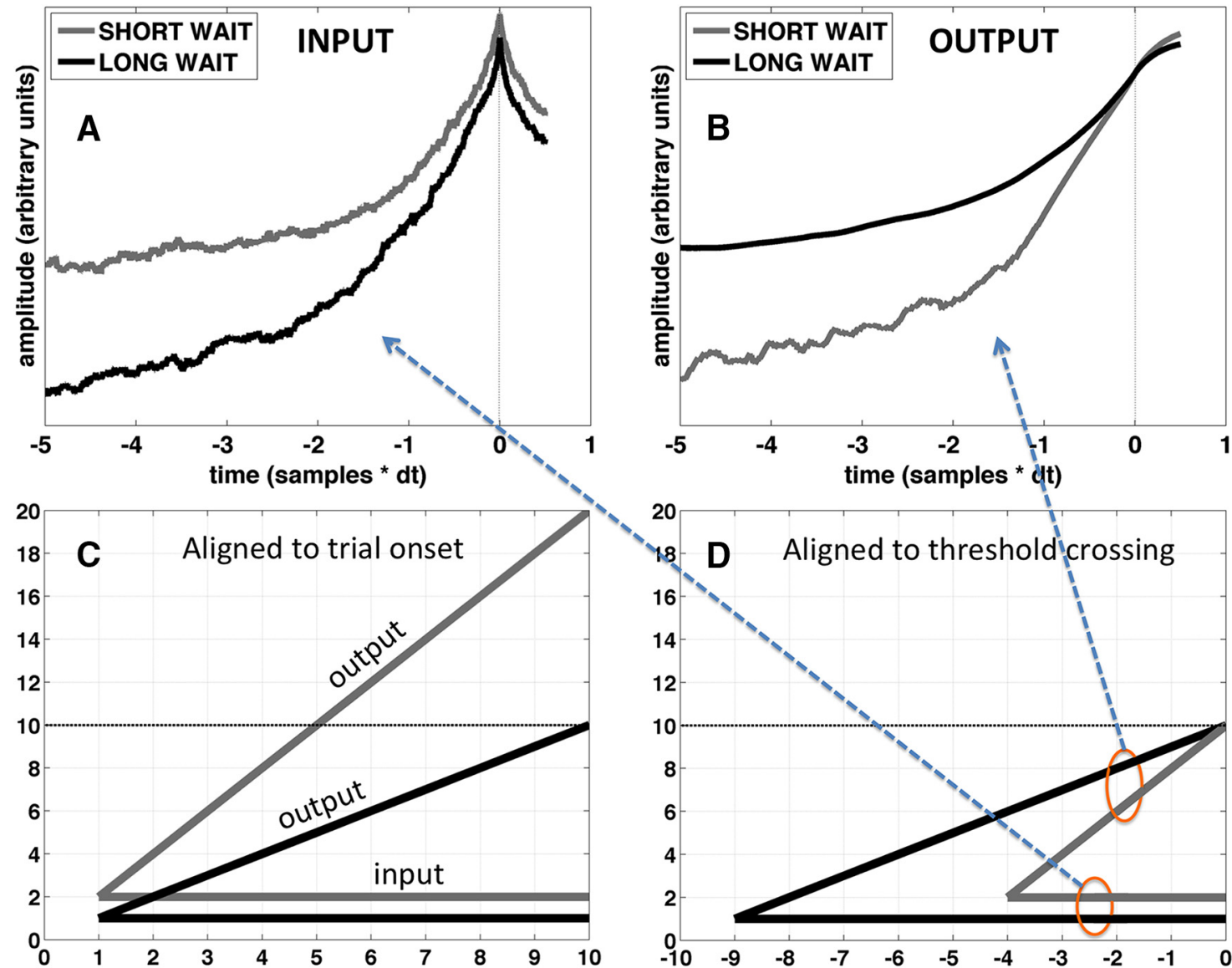

Figure 5. Predicting the shape of the RP as a function of waiting time. A, Average stochastic input to the accumulator, time aligned to first crossing times in the output, separately for trials with a long wait (upper 33rd percentile; black line) and for trials with a short wait (lower 33rd percentile; gray line). B. Same as $\boldsymbol{A}$ but for the output of the accumulator. The early tail of the output on "short wait" trials is noisier than the rest because of missing data: on trials with a short wait, often the climb to the threshold was shorter than the epoch length. $\boldsymbol{C}$, Schematic depiction of the input and output for constant input, time aligned to the beginning of the trial. On trials with a short wait, the input is greater and the output rises more quickly to the threshold. $\boldsymbol{D}$, Same as $\boldsymbol{C}$, but time aligned to the threshold crossing. Notice that when time is aligned to the threshold crossing the relationship between input and output becomes reversed. This helps to intuitively explain the reversal in the relationship between the predicted shape of the RP for long- and short-wait time trials. Parameters used for $\boldsymbol{A}, \boldsymbol{B}: \beta=1.4, \mathrm{I}=0.1, \mathrm{k}=0.6$, and threshold $=0.1256$. However, the relationship between predicted RPs for short versus long wait times (reversal of amplitude relationship for input versus output) remained qualitatively the same regardless of the specific parameters used, as long as $\beta$ was $>\sim 0.5$. Regarding $\boldsymbol{A}, \boldsymbol{B}$, note that, because the epochs are time locked to threshold crossings in the output, only the outputs $(\boldsymbol{B})$ are guaranteed to reach the same amplitude at $t_{(0)}$. The two curves in $\boldsymbol{A}$ do not necessarily have to reach the same amplitude at $t_{(0)}$, because these are the average inputs to the accumulator. The inputs for long and short waits in $\boldsymbol{C}, \boldsymbol{D}$ are set to 1 and 2, respectively, for illustrative purposes, so that the slope of their respective outputs will be 1 and 2 . Note that this overly simplified schematic is only intended to describe the relationship between the input and output but not their shape.

translates into a lower initial amplitude, when time aligned to the first crossing time. Whereas trials with a longer waiting time will tend to have a more gradual, shallow slope, and thus a higher amplitude when time aligned to the first crossing time. The input to the accumulator, on the other hand, will tend to have a higher amplitude throughout the epoch for trials with a short waiting time (the higher its amplitude the sooner its integral will cross a given threshold value). This relationship is explained schematically in Figure $5 C, D$, which illustrates the relationship for the simplest case where the input is constant and the output is linear. Note that this overly simplified schematic is only intended to describe the relationship between the input and output but not their shape.

\section{Prediction 2: correlation between waiting time and W time}

As mentioned in Materials and Methods, I assume that the temporal delay between the crossing of the advance warning threshold and the main activation threshold contributes to the subjective estimate of 'W' time (Fig. 2). Thus, if the trajectory of the decision variable is relatively steep, then that temporal delay will be relatively short, and so ' $W$ ' time will be later in time (closer in time to the onset of movement). On the other hand, if the trajectory of the decision variable happens to be relatively gradual, then the temporal delay will be longer and ' $W$ ' time will be earlier (farther back in time from the onset of movement). Therefore, the prediction is that waiting time should be 


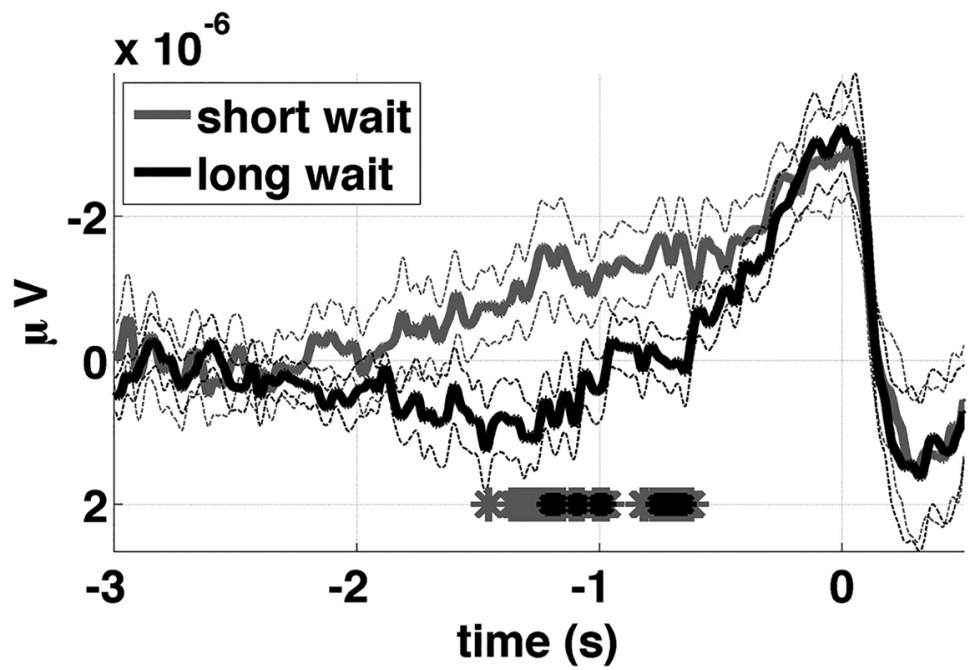

Figure 6. The shape of the RP as a function of waiting time. Average empirical RP (sign reversed for easier comparison with model predictions) at electrode C1 for trials with short (gray line) and long (black line) waiting times. Thin dashed lines show standard error of the mean. Stars at the bottom of the axis mark time points where the difference between the two was significant at $p<0.05$ (gray stars) and $p<0.01$ (black stars); $p<0.01$ for the mean amplitude over the range -1.5 to $-0.5 \mathrm{~s}$ (two-tailed signed rank test).

negatively correlated with ' $W$ ' time, with longer waiting times predicting earlier ' $W$ ' times and vice versa (Fig. 7). Remarkably, this prediction is confirmed, both in the pooled data from all subjects (Fig. 8; Pearson's correlation coefficient: $r=-0.116, p=0.0003$; slope of regression line: $b=-0.012)$, and in the aggregate of individual correlations across subjects (Fig. 9; $p=0.0009$, two-sided Wilcoxon signed rank test on the $r$ 's from each individual subject, $n=14 ; r$ was negative for 13 of 14 subjects, $p=$ 0.0009 binomial).

The parameters used to generate the above prediction were the same as those used for fitting the data and predicting the shape of the RP: $\beta=1.4, I=0.1, k=0.6$, and threshold $=0.1256$. The prediction was robust to small changes in the parameters. Most importantly the prediction only held for $\beta$ more than $\sim 1.2$, i.e., this prediction depends on the stochastic input being pink. Thus, allowing $\beta$ to vary revealed a novel prediction that would not have been revealed otherwise.
The advance warning threshold may be thought of as analogous to the processing of an "efference copy" by an internal forward model (Wolpert, 1997). When the prediction threshold is crossed, information is generated indicating that a specific movement is just about to happen with high probability. After the movement is completed, this information informs the subsequent subjectivelyestimated ' $W$ ' time. Note again that I do not assume that the predictive signal uniquely determines ' $W$ ' time, but only that it informs ' $W$ ' time. There is strong evidence that neural information from both before and after movement onset can influence ' $W$ ' time, and thus that the brain likely makes use of information from both before and after movement onset in making this particular judgment (Lau et al., 2007; Banks and Isham, 2009; Douglas et al., 2015). Here, I assume that a premovement prediction about an upcoming movement (and its reafferent consequences) is at least part of the information that contributes to the brain's post hoc estimate of 'W' time.

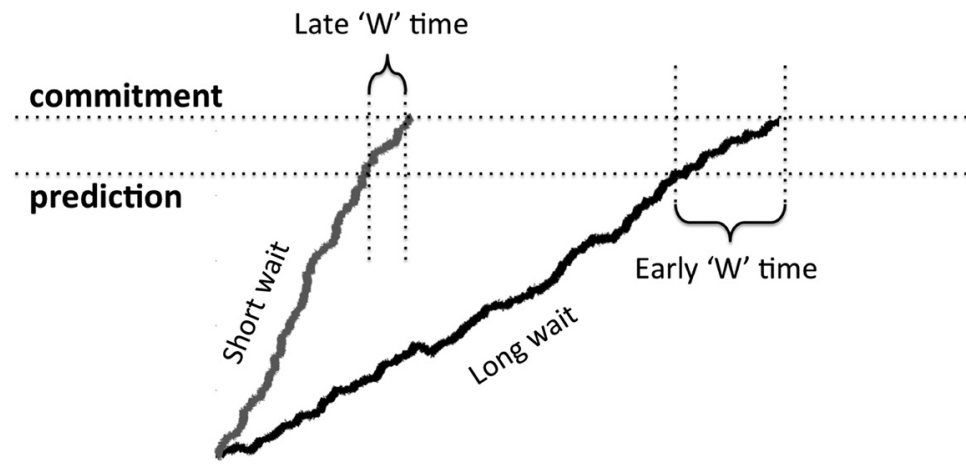

Figure 7. Predicting 'W' time as a function of waiting time. Schematic showing how 'W' time is predicted to vary as a function of waiting time. On trials with a short waiting time, the slope of the decision variable is steeper (gray line) than on trials with a long waiting time (black line). A steeper slope means that the interval between the crossing of the two thresholds will be shorter, so 'W' time will be closer in time to the onset of movement (smaller in absolute value). For trials with a more gradual slope of the decision variable (black line) the reverse is true, 'W' time will be further back in time from the onset of movement and larger in absolute value. 

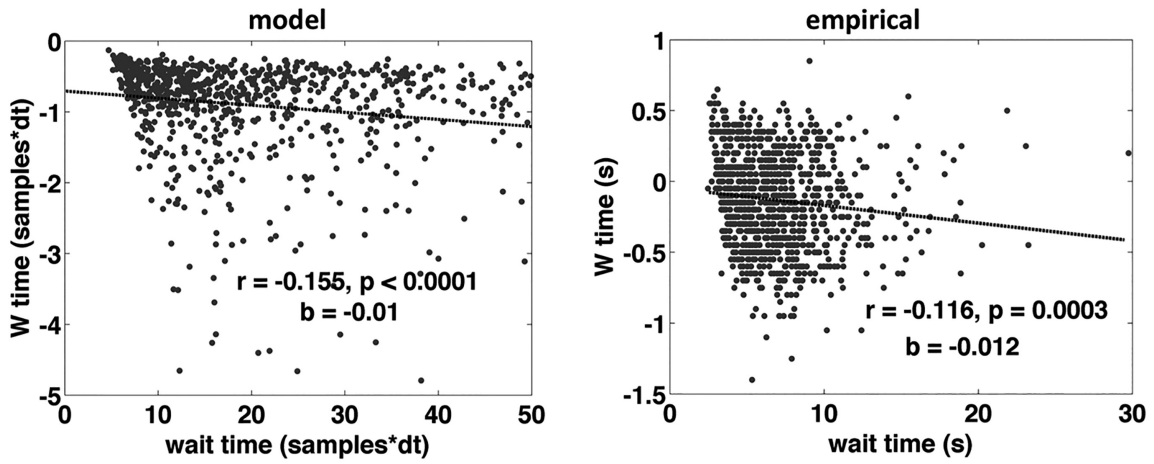

Figure 8. 'W' time as a function of waiting time aggregate. Correlation between waiting time and 'W' time for simulated data from the model (left) and for the empirical data from all subjects (right; each dot is one trial from one subject). Although there is noise in the model, the model data are bounded by zero at the top, because there is no noise in the estimation of ' $W$ ' time, it is strictly earlier than movement time and is "reported" exactly as is. In reality there is a lot of variance across trials and across subjects in the reporting of ' $W$ ' time, and this is evident in the panel on the right. Combining the data from all subjects can be problematic, because differences between subjects and differences within subject are confounded, so in Figure 9, I present the correlations separately for each subject.

As mentioned before, to guard against the possibility that this correlation might be driven by the difference in mean waiting time for the classic and interruptus tasks (recall that noninterrupted trials from the interruptus task were combined with data from the classic task), I tested the pooled correlation separately for the data from the classic task and the data from the interruptus task. The correlation was negative and highly significant in both data sets (classic task: $r=-0.1159, p<0.001$; interruptus task: $r=-0.1156, p<0.001)$, thus arguing against this possible interpretation.

\section{Discussion}

Here, I explored the behavior of an accumulator model when the power spectrum of the input noise was allowed to vary from white (the standard for such models) to pink, i.e., allowing for varying levels of temporal autocorrelation in the noise. The $1 / f$ exponent $(\beta)$ of the noise spectrum thus becomes a parameter of the model, which can take values in the range $0 \leq \beta<3$. Previous work has looked at stochastic differential equations that can generate autocorrelated noise in their output with a variable 1/f exponent (Ruseckas and Kaulakys, 2010), but this is different

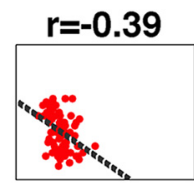

$r=-0.33$

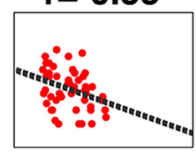

$r=-0.32$
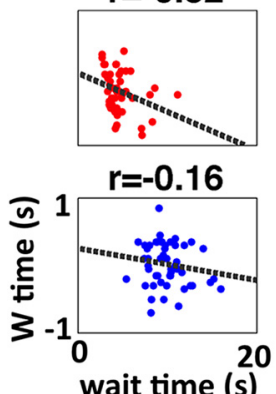

wait time (s) $r=-0.18$

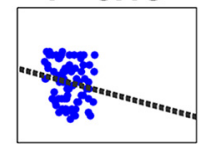

$r=-0.03$

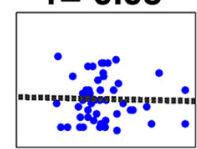

$r=-0.18$

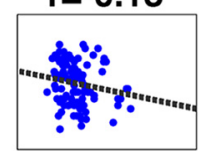

$r=0.06$

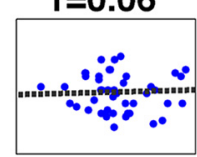

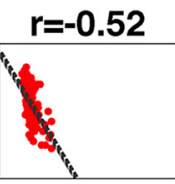

$r=-0.05$

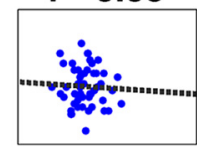

$r=-0.33$

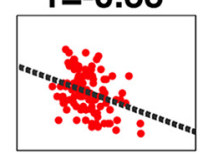

overall $p=0.0009$

Wilcoxon

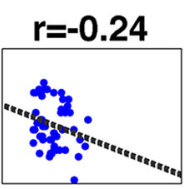

$r=-0.14$

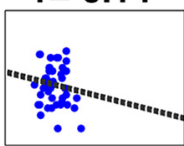

$r=-0.05$

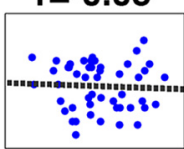

Figure 9. 'W' time as a function of waiting time per subject. Correlation between waiting time and 'W' time for the empirical data grouped by subject. The horizontal axis is the waiting time (from 0 to $20 \mathrm{~s}$ ) and the vertical axis is 'W' time in seconds with respect to movement onset. Data are shown in red if the correlation is individually significant at $p<0.05$. When the $r$ values for all subjects are submitted to a Wilcoxon signed rank test the effect is significant at $p<0.001$. Also, the probability of 13 subjects (out of 14 ) individually exhibiting a negative correlation is 0.0009 (binomial test). 
from the present work which used autocorrelated noise in the input to the process (in place of the Gaussian white noise used by convention).

With the $1 / f$ exponent $(\beta)$ of the input noise as a parameter, I tested the possibility that the canonical RP reflects the average event-locked stochastic input to an accumulator rather than its average output. I first showed that the model, under the RP-as-input assumption, was able to fit the data and then tested a prediction regarding the shape of the RP. Finally, I also tested a prediction regarding 'W' time that depends on the input noise being autocorrelated.

Intuitively one might expect that, from the perspective of a scalp electrode, the output of a neural accumulator might be too faint to be detected, because it might be computed by a small population of neurons (compare O'Connell et al., 2012). At the same time, the stochastic input to the decision process should be at least as readily measured at the scalp if it is shared among a large number of neurons spanning a functional network (Mantini et al., 2007). Whatever the case may be, the model provided a way to distinguish between these two possibilities by generating opposing predictions depending on the substrate (input or output) of the observed average (Fig. 5).

The model also incorporated a second "warning" threshold, slightly lower than the primary activation threshold, such that when the lower threshold is crossed the probability of soon crossing the primary activation threshold is high (Fig. 7). This lower threshold allows the model to account for the estimated time of the subjective urge to move ('W' time) in terms of the delay between the crossing of the two thresholds. This is clearly an oversimplification of the true state of affairs: prior data have implicated factors from both before and after movement onset in the subjective estimation of 'W' time (Sirigu et al., 2004; Lau et al., 2007; Banks and Isham, 2009; Desmurget et al., 2009; Douglas et al., 2015). However, the model is only intended to account for one such factor, originating before movement, plausibly the generation of a forward model (Wolpert, 1997). There has been a great deal of controversy surrounding the meaning of ' $W$ ' time and its relationship to the onset of intention (for review, see Maoz et al., 2015).

Note that the argument here is not that pink noise is superior to white noise in stochastic accumulator models. Rather it is an argument in favor of allowing $\beta$ to vary (as it does in real neural systems) in accumulator models of decision-making. The resulting value for $\beta$ that is suggested by the model could in principle be near zero (i.e., white noise) or it could be roughly between 1 and 2 (pink noise). The point is that having $\beta$ as a parameter adds a new dimension to the model allowing it to account more fully for the neural data, by fitting its spectral properties. One advantage of doing so is that the stochastic input may then behave in a way that is amenable to modeling, e.g. the the RP-as-input variant of the model can only fit the data when $\beta$ is non-zero. Another advantage is that with variable $\beta$, we can account for properties of the data that were previously overlooked, potentially leading to new predictions.
Allowing the spectral properties of the input noise to vary here led to two novel predictions. The first prediction was that the shape of the RP should vary as a function of the time elapsed between the beginning of the trial and the onset of the self-initiated movement (the waiting time). The relationship between the shape of the RP and waiting time was reversed depending on whether the RP was modeled as the average input to or average output from the accumulator (Fig. 5) thus constituting a strong test with which to adjudicate between these two possibilities. The second prediction was that the estimated time of the subjective urge to move, with respect to movement onset, should be negatively correlated with the waiting time, being earlier in time (with respect to movement onset) for longer waiting times, and vice versa. Both of these predictions were confirmed, lending support to the hypotheses encapsulated in the model. The results are important because they drive a wedge between two different possible (and plausible) interpretations of the RP: (1) as the average event-locked stochastic input to an integrationto-bound process, or (2) as the average event-locked output from an integration-to-bound process. Both results point to the former interpretation.

Note that Caspar and Cleeremans (2015) found that the amplitude of the RP was lower among subjects with late 'W' times ("short W group") compared to subjects with early 'W' times ("Iong W group"), whereas Haggard and Eimer (1999) found the opposite. These studies did not group their data according to waiting time as I have done here, but one can infer the following: If longer waiting times are associated with both earlier ' $W$ ' times and a lower-amplitude early RP, then early ' $W$ ' times may be associated with a lower-amplitude early RP. This is broadly consistent with Caspar and Cleeremans (2015), although the difference here was in the early RP, whereas these two prior studies report a difference in the amplitude of the RP overall. Note also that the results of both Haggard and Eimer (1999) and Caspar and Cleeremans (2015) depend on the choice of baseline which has recently been a topic of debate (Khalighinejad et al., 2018). I did not apply any baseline correction to the EEG data reported here.

The hypotheses tested in the present study are based on two premises. One is that, when movements are made spontaneously and voluntarily, a subset of neurons in premotor areas behave like inputs to an accumulator while other neurons behave like outputs (Murakami et al., 2014). This first premise raises the question of which subpopulation of neurons is responsible for the RP, as captured from the perspective of a scalp electrode, or other mass-action recording modality. The second premise is that real biological noise in the brain is not white, but rather has (approximately) a 1/f spectral profile. Although we have known about the RP for a long time, attempts to model it mechanistically have come about only recently (Schurger et al., 2012). These two premises expand the horizon of stochastic accumulator models, especially in the low-SNR regime, and add a new dimension to the kinds of hypotheses that can be derived from such models. 
The model developed here accounts for both the shape of the RP and W time in terms of a third variable, the time spent waiting to produce a movement. To date, this variable has been given very little attention in the literature, but was predicted to be a factor by the model. To the extent that the waiting time in a self-initiated movement task can be considered analogous to the reaction time in a perceptual decision-making task, we can then consider some of the parallels between them. From the point of view of the accumulator model, both reaction time and waiting time reflect the very same thing: the time it takes for the integral of evidence plus noise to reach a decision boundary. Both produce a gamma distribution, and both exhibit a positive linear relationship between the standard deviation and the mean (Schurger et al., 2012).

The simplifying assumption of Gaussian white noise in accumulator models has held strong for decades, and such models have proven themselves very capable of accounting for behavioral and neural data (Ratcliff, 1978; Ratcliff et al., 1999; Gold and Shadlen, 2001; Usher and McClelland, 2001; Ratcliff et al., 2004; Gold and Shadlen, 2007; Ratcliff and Starns, 2009, 2013). This begs the question of why we should further complicate things by introducing varying levels of autocorrelation in the input noise. The answer is simple: stochastic fluctuations in neural systems tend to be temporally autocorrelated (Destexhe et al., 2003; Rudolph and Destexhe, 2003; Henrie and Shapley, 2005; Garcia-Perez et al., 2007; Milstein et al., 2009; Van De Ville et al., 2010; He, 2011). A real neural integrator, given that it must integrate over fluctuations originating elsewhere in the brain, will likely integrate over autocorrelated fluctuations, and if so then the level of autocorrelation will likely vary between different cognitive states and between different individuals $(\mathrm{He}$ et al., 2010). Thus, the level of autocorrelation (1/f exponent) in neural time series is a meaningful and functionally relevant physiological variable that can be accounted for in models of decision-making by allowing the spectral properties of the stochastic input to vary.

While the use of simulated $1 / f$ noise as input to the accumulator yields a very good fit to the data (wait-time distribution and RP) there might be other ways to accomplish this. I was able to achieve a moderately good fit to the data using low-pass filtered white noise, although the best fit was still significantly poorer than that achieved using simulated 1/f noise. Also, the (log-log) slope of the resulting spectrum was far from that observed in the empirical data $(\sim 3.0$ for low-pass filtered noise vs $\sim 1.4$ for simulated $1 / f$ noise). So, while it might be possible to fit the wait-time distribution and/or RP using low-pass filtered white noise as input, the spectrum of the resulting input time series is a very poor match to that observed empirically. Thus, one key advantage of using simulated $1 / f$ noise versus low-pass filtered white noise is that it can account not only for the behavior and event-related potential, but also for the spectral properties of the EEG data, offering a more complete representation of the data. Still, no claim is made here as to whether or not true power-law noise is required. What is required, at a minimum, is that the stochastic input time series are tempo- rally autocorrelated and that the degree of autocorrelation can be varied parametrically. The technique used here to generate simulated 1/f noise (see Materials and Methods) offers a means of doing that.

Accumulator models are commonly viewed as operating at a higher level of abstraction than neural models. Nevertheless, aspects of such models are commonly mapped onto neural phenomena (Gold and Shadlen, 2007). The output of the accumulator ( $x$, the decision variable) is commonly taken to represent the firing rate of neurons involved in decision-making, and the constant input $(I)$ is taken to represent the sensory evidence in the form of firing rates of sensory perceptual neurons that synapse, directly or indirectly, onto the decision neurons. The noise term on the other hand is not typically considered to map directly onto a specific neural phenomenon, but rather accounts for variability within and across trials. Here, I offer a more explicit treatment of what the noise term reflects: it reflects stochastic variability in neural activity originating from elsewhere in the brain, and as such perhaps should be modeled as having the same power spectrum as real neural noise. This spectrum is known to be pink rather than white.

One might argue that, although the use of autocorrelated noise in the model is more biologically realistic, it may add relatively little in terms of accounting for and helping to explain the data. As mentioned previously, standard accumulator models, with Gaussian white input noise, have been widely used in the study of perceptual decision-making and reaction time paradigms and have proven very effective in accounting for neural and behavioral data. While this may be true, the fact that the predictions made here emerge from the model only when the input noise is autocorrelated lends credence to the argument that the spectral properties of the noise used in such models does matter. Further research will be needed to bear this out, but intuitively it seems that it will likely matter most when the imperative (the drift term) is weak relative to the noise, i.e., in the context of decision-making under uncertainty.

\section{References}

Banks WP, Isham EA (2009) We infer rather than perceive the moment we decided to act. Psychol Sci 20:17-21. CrossRef Medline

Caspar EA, Cleeremans A (2015) "Free will": are we all equal? A dynamical perspective of the conscious intention to move. Neurosci Conscious 2015:niv009.

Desmurget M, Reilly KT, Richard N, Szathmari A, Mottolese C, Sirigu A (2009) Movement intention after parietal cortex stimulation in humans. Science 324:811-813. CrossRef Medline

Destexhe A, Rudolph M, Paré D (2003) The high-conductance state of neocortical neurons in vivo. Nat Rev Neurosci 4:739-751. CrossRef Medline

Douglas ZH, Maniscalco B, Hallett M, Wassermann EM, He BJ (2015) Modulating conscious movement intention by noninvasive brain stimulation and the underlying neural mechanisms. J Neurosci 35:7239-7255. CrossRef Medline

Ganos C, Asmuss L, Bongert J, Brandt V, Münchau A, Haggard P (2015) Volitional action as perceptual detection: predictors of conscious intention in adolescents with tic disorders. Cortex 64:4754. CrossRef Medline 
Garcia-Perez E, Mazzoni A, Torre V (2007) Spontaneous electrical activity and behavior in the leech Hirudo medicinalis. Front Integr Neurosci 1:1:8. CrossRef

Gold JI, Shadlen MN (2001) Neural computations that underlie decisions about sensory stimuli. Trends Cogn Sci 5:10-16. Medline

Gold JI, Shadlen MN (2007) The neural basis of decision making. Annu Rev Neurosci 30:535-574. CrossRef Medline

Haggard P, Eimer M (1999) On the relation between brain potentials and the awareness of voluntary movements. Exp Brain Res 126: 128-133. Medline

He BJ (2011) Scale-free properties of the functional magnetic resonance imaging signal during rest and task. J Neurosci 31:1378613795. CrossRef Medline

He BJ, Zempel JM, Snyder AZ, Raichle ME (2010) The temporal structures and functional significance of scale-free brain activity. Neuron 66:353-369. CrossRef Medline

Henrie JA, Shapley R (2005) LFP power spectra in V1 cortex: the graded effect of stimulus contrast. J Neurophysiol 94:479-490. CrossRef Medline

Jung TP, Makeig S, Humphries C, Lee TW, McKeown M, Iragui V, Sejnowski TJ (2000) Removing electroencephalographic artifacts by blind source separation. Psychophysiology 37:163-178. Medline

Khalighinejad N, Schurger A, Desantis A, Zmigrod L, Haggard P (2018) Precursor processes of human self-initiated action. Neuroimage 165:35-47. CrossRef Medline

Kornhuber HH, Deecke L (1965) Hirnpotentialänderungen bei Willkürbewegungen und passiven Bewegungen des Menschen: Bereitschaftspotential und reafferente Potentiale. Pflügers Archiv 284:1-17. CrossRef

Lau H, Rogers RD, Passingham RE (2007) Manipulating the experienced onset of intention after action execution. J Cogn Neurosci 19:81-90. CrossRef Medline

Libet B, Gleason CA, Wright EW, Pearl DK (1983) Time of conscious intention to act in relation to onset of cerebral-activity (readinesspotential). The unconscious initiation of a freely voluntary act. Brain 106:623-642. CrossRef

Mantini D, Perrucci MG, Del Gratta C, Romani GL, Corbetta M (2007) Electrophysiological signatures of resting state networks in the human brain. Proc Natl Acad Sci USA 104:13170-13175. CrossRef

Maoz U, Mudrik L, Rivlin R, Ross I, Mamelak A, Yaffe G (2015) On reporting the onset of the intention to move. In: Surrounding free will: philosophy, psychology, neuroscience (Mele A, ed), pp 184202. New York: Oxford University Press.

Milstein J, Mormann F, Fried I, Koch C (2009) Neuronal shot noise and Brownian 1/f(2) behavior in the local field potential. PLoS One 4. CrossRef

Murakami M, Vicente MI, Costa GM, Mainen ZF (2014) Neural antecedents of self-initiated actions in secondary motor cortex. Nat Neurosci 17:1574-1582. CrossRef Medline
O'Connell RG, Dockree PM, Kelly SP (2012) A supramodal accumulation-to-bound signal that determines perceptual decisions in humans. Nat Neurosci 15:1729-1735.

Oostenveld R, Fries P, Maris E, Schoffelen JM (2011) FieldTrip: open source software for advanced analysis of MEG, EEG, and invasive electrophysiological data. Comput Intell Neurosci 2011:156869. CrossRef

Pereda E, Gamundi A, Rial R, González J (1998) Non-linear behaviour of human EEG: fractal exponent versus correlation dimension in awake and sleep stages. Neurosci Lett 250:91-94. Medline

Ratcliff R (1978) A theory of memory retrieval. Psychol Rev 85:59_ 108. CrossRef

Ratcliff R, Starns JJ (2009) Modeling confidence and response time in recognition memory. Psychol Rev 116:59-83. CrossRef Medline

Ratcliff R, Starns JJ (2013) Modeling confidence judgments, response times, and multiple choices in decision making: recognition memory and motion discrimination. Psychol Rev 120:697719. CrossRef Medline

Ratcliff R, Van Zandt T, McKoon G (1999) Connectionist and diffusion models of reaction time. Psychol Rev 106:261-300. Medline

Ratcliff R, Gomez P, McKoon G (2004) A diffusion model account of the lexical decision task. Psychol Rev 111:159-182. CrossRef Medline

Rudolph M, Destexhe A (2003) Characterization of subthreshold voltage fluctuations in neuronal membranes. Neural Comput 15: 2577-2618. CrossRef Medline

Ruseckas J, Kaulakys B (2010) 1/f noise from nonlinear stochastic differential equations. Phys Rev E 81:031105. CrossRef

Schmidt S, Jo H-G, Wittmann M, Hinterberger T (2016) 'Catching the waves' - slow cortical potentials as moderator of voluntary action. Neurosci Biobehav Rev 68:639-650. CrossRef Medline

Schurger A, Sitt J, Dehaene S (2012) An accumulator model for spontaneous neural activity prior to self-initiated movement. Proc Natl Acad Sci USA 109:E2904-E2913. CrossRef

Shibasaki H, Hallett M (2006) What is the Bereitschaftspotential? Clin Neurophysiol 117:2341-2356. CrossRef Medline

Sirigu A, Daprati E, Ciancia S, Giraux P, Nighoghossian N, Posada A, Haggard $P$ (2004) Altered awareness of voluntary action after damage to parietal cortex. Nat Neurosci 7:80-84. CrossRef

Usher M, McClelland JL (2001) The time course of perceptual choice: the leaky, competing accumulator model. Psychol Rev 108:550592.

Van De Ville D, Britz J, Michel C (2010) EEG microstate sequences in healthy humans at rest reveal scale-free dynamics. Proc Natl Acad Sci USA 107:18179-18184. CrossRef Medline

Wen H, Liu Z (2016) Separating fractal and oscillatory components in the power spectrum of neurophysiological signal. Brain Topogr 29:13-26. CrossRef Medline

Wolpert DM (1997) Computational approaches to motor control. Trends Cogn Sci 1:209-216. CrossRef Medline 